\section{P-221 DEVELOPMENT OF ANNUAL GP REFERRAL REPORT TO INCREASE ENGAGEMENT, AWARENESS AND REFERRALS}

Stephen Roberts. North Devon Hospice, Barnstaple, UK

10.1136/bmjspcare-2019-HUKNC.243

North Devon Hospice recognised the important role that GPs play in ensuring that patients and their loved ones benefit from the full suite of services that the hospice provides. North Devon Hospice also recognises the workload of GPs means that throughout a given year their awareness of hospice services, particularly for non-cancer patients, may vary significantly. Ultimately this leads to unmet need.

To address this we sought a solution which enabled engagement with the GPs above and beyond the time constrained, patient focused meetings that our clinical nurse specialists have with all of the GPs across North Devon. We also felt that it is not the responsibility of the CNSs to hold the entire relationship management with each GP Practice.

Against this backdrop we developed the annual GP referral report for each GP Practice. This report included:

- Top 10 referrers by GP Practice: a league table of where each GP practice sits against others on the number of referrals;

- Primary reason for referral;

- Secondary services that patients then accessed following initial referral: thereby demonstrating the extra support that is wrapped around the patient once they have been referred;

- List of the GPs in each practice who made the referral;

- Diagnosis of patients split by cancer $\mathrm{v}$ non-cancer: opportunity to highlight that the hospice is not just about cancer;

- Number of patients who received our Hospice to Home service.

The results were very tangible. We saw between a $10 \%-$ $30 \%$ increase on our referrals per practice and then a higher referral for non-cancer conditions in the three months after the report was issued.

We have now timed the publishing of the report to capture the gap in the year when we as a hospice are not active in each practice either through education sessions or through the annual visit by the CEO, Medical Director and Director of Care.

\section{P-222 IMPLEMENTING A COMMUNITY CLINICAL NURSE SPECIALIST TELEPHONE TRIAGE: A MIXED METHODS EVALUATION}

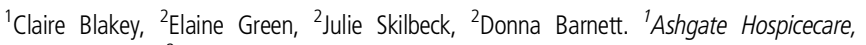
Chesterfield, UK; ${ }^{2}$ St.Luke's Hospice, Sheffield, UK

\subsection{6/bmjspcare-2019-HUKNC.244}

Background There is evidence that using telephone triage to enable access to specialist palliative care services can enhance the assessment of patient need, provide rapid access to specialist services and manage increasing demand (Sutherland \& Stananought, 2011).

Audit of patient referrals to our Community Palliative Care Specialist Nurse Service highlighted variability in patient waiting times before accessing the service. We introduced a CNS telephone triage service in June 2017 and are currently evaluating its impact.
Aims To evaluate the extent to which the telephone triage service provides a co-ordinated, timely and responsive service for patients with complex palliative and end-of-life care needs. Methods Phase 1 Feb-March 2018: Literature review, logic model development. Phase 2 Apr-Sept 2018: Interviews $(n=11)$ and focus groups $(n=2)$ with key stakeholders. Phase 3 Oct-Jan 2019: Online survey with referrers to the service. Phase 4 Mar-June 2019: Case mix analysis of patients allocated Red (Unstable) Amber (Deteriorating) Green (Stable) status at triage; followed for 4 weeks $(n=120)$. We counted: time to first visit, number of visits, telephone calls, OACC measures (Witt, de Wolf-Linder, Dawkins, Daveson et al., 2013).

Results Waiting time from referral to triage to start of CNSs' care has reduced (2.5 days to 1.4 days average). Patients identified as Red (Unstable) visited within 24/48 hours. Key stakeholders consider that triage has contributed to the delivery of a more timely and responsive service for patients. Referrers commented that the referral template duplicated data on SystmOne. Early case mix analysis indicates minimal differences between the number of visits per patients identified as Urgent (Red) or Deteriorating (Amber).

Conclusion Our evaluation findings demonstrate that telephone triage improves patient access to our Community Palliative Care Specialist Nurse Service. We learnt that the model of triage has to be robust and flexible to withstand unforeseen workforce issues; we are currently exploring alternative approaches.

\section{P-223 HOSPICE SUPPORT ASSISTANTS}

Anne-Marie Crook, Kelly McManus, Tracy Rhodes, Leone Beet. St Catherine's Hospice, Preston, UK

\subsection{6/bmjspcare-2019-HUKNC.245}

Background The Hospice Support Assistant (HSA) role is a new and innovative idea to reach as many patients as possible in the community who may need psychological support and non-pharmacological symptom management. This is a three year pilot project funded by the hospice with one year funding provided by the local CCGs.

Aims Initially the role was to be based in the community supporting patients in a non-pharmacological way, focusing on Fatigue, Anxiety and Breathlessness, also to offer psychological support to family/loved ones as well as the patient. We have adapted and used a Goal Attainment Scaling system to measure patients' ability and needs throughout our intervention.

Method Data is currently being extracted, looking at referrals made initially, how many visits made, what interventions are used and patient/relative feedback forms (iWantGreatCare) also if this increased the number of patients achieving their preferred place of care/death.

Implementation Since the role went live the realisation has been that there is a lot more involved than originally thought e.g. social intervention, listening, sign-posting, family/loved one involvement, distraction therapy, mental stimulation, liaising with CNSs and other hospice teams as well as external support agencies.

Evaluation The role is developing well and the caseloads are increasing, feedback from patients so far is positive. Training is ongoing with upcoming opportunities to further build the knowledge and skills already gained. 